\title{
RENDIMIENTO DEL ULTRASONIDO EN EL DIAGNÓSTICO DE ACRETISMO PLACENTARIO EN EL INSTITUTO NACIONAL MATERNO PERINATAL
}

\author{
Erasmo Huertas Tacchino 1,a, b
}

\begin{abstract}
RESUMEN
Objetivos. Determinar la sensibilidad y especificidad del ultrasonido en la detección prenatal del acretismo placentario. Materiales y métodos. Diseño: Estudio observacional analítico retrospectivo Lugar: Unidad de Medicina Fetal. Instituto Nacional Materno Perinatal. Lima-Perú. Participantes: Gestantes que acudieron a la Unidad entre el 02 de enero y el 31 de diciembre del 2012, para realización de ecografía obstétrica por sospecha de acretismo. Se revisaron las historias clínicas de 93 pacientes evaluadas mediante ecografía durante el segundo y tercer trimestre buscando signos de acretismo, comparando luego con los hallazgos durante el parto y la anatomía patológica. Resultados. La sensibilidad del diagnóstico ecográfico de acretismo placentario es de $90.9 \%$ y la especificidad de $99 \%$. La edad gestacional al momento del diagnóstico ecográfico fue en el $50 \%$ de casos entre las 32 y 36 semanas, siendo el diagnóstico más precoz el realizado a las 25 semanas. En el $100 \%$ de los casos el diagnóstico ecográfico de acretismo estuvo asociado al hallazgo de placenta previa y en el $70 \%$ de culminó en cesárea + histerectomía Conclusiones. El ultrasonido es una herramienta útil en el diagnóstico prenatal de acretismo placentario. Debe sospecharse en toda paciente con diagnóstico ecográfico de placenta previa.
\end{abstract}

Palabras Clave. Placenta acreta; Ultrasonido; Diagnóstico prenatal (Fuente: DeCS BIREME).

\section{PERFORMANCE OF THE ULTRASOUND IN THE DIAGNOSIS OF PLACENTARY ACRETISM AT THE PERINATAL MATERNAL NATIONAL INSTITUTE}

\begin{abstract}
Objectives. To determine sensibility and specificity of ultrasound in prenatal detection of placenta accreta and to correlate with obstetric outcomes. Material and methods. Design: Observational, analytic and retrospective study. Patients: Pregnant women that went into Fetal Medicine Unit between January 1st and December 31st 2012 to perform an obstetric ultrasound because placenta accreta was suspected. Clinical records of 93 patients that were performed ultrasound between second and third trimester looking for ultrasound signs of placenta accreta were reviewed, the results were then compared with findings at delivery and in pathology. Results. Ultrasound sensibility for diagnosis of Placenta accreta is $90 \%$ and specificity is $99 \%$. Gestational age at the moment of diagnosis in $50 \%$ of cases was between 32 and 36 weeks, and the earliest age was 25 weeks. Placenta previa was associated in $100 \%$ of cases of Placenta accreta and in $70 \%$ pregnancy ended in cesarean section + hysterectomy Conclusions. Ultrasound is a useful tool in prenatal diagnosis of Placenta accreta. The condition must be suspected in all patients with placenta previa by ultrasound.
\end{abstract}

Key words: Placenta Accreta; Ultrasound; Prenatal diagnosis(Source: MeSH NLM).

\section{INTRODUCCIÓN}

El acretismo placentario representa un espectro de anomalías de la placentación, caracterizado por una adherencia anormal de ésta a la pared uterina. hoy en día se le define como placenta anormalmente invasiva y comprende la placenta acreta, increta y percreta ${ }^{1}$.

Ocurre en 1 cada 533 partos y su incidencia se ha incrementado 10 veces en los últimos 50 años, en relación directa con el incremento de la tasa de cesáreas ${ }^{2,3}$. Se considera como una complicación severa del embarazo que puede asociarse con hemorragia intra parto y post parto masiva y potencialmente letal y su detección prenatal podría jugar un papel fundamental en el propósito de disminuir el riesgo de morbimortalidad materna. En la literatura internacional existen varios reportes epidemiológicos de esta patología, sin embargo, en nuestro medio no existen reportes de su incidencia a nivel nacional solo existen 3 publicaciones al respecto con una antigüedad que supera los 10 años $^{4-6}$. Tampoco existen en nuestras medias publicaciones que evalúen la eficacia del diagnóstico prenatal mediante ultrasonido a pesar de su uso masivo en la práctica clínica.

\footnotetext{
Médico Especialista en Ginecología y Obstetricia

a Unidad de Medicina Fetal, Instituto Nacional Materno Perinatal, Lima-Perú.

Departamento Académico de Ginecología, Facultad de Medicina, Universidad Nacional Mayor de San Marcos, Lima-Perú
}

Citar como: Huertas E. Rendimiento del ultrasonido en el diagnóstico de acretismo placentario en el Instituto Nacional Materno Perinatal. Rev Peru Investig Matern Perinat 2019; 8(1):13-6.

DOI: https://doi.org/10.33421/inmp.2019134

Recibido: 07-03-19 Aprobado: 27-03-19 
El objetivo general fue determinar la sensibilidad, especificidad, valor predictivo positivo y valor predictivo negativo del ultrasonido en el diagnóstico de acretismo placentario en el Instituto Nacional Materno Perinatal (INMP).

\section{MATERIALES Y MÉTODOS}

Se realizó un estudio observacional, analítico, retrospectivo de todas las pacientes que acudieron al Servicio de Medicina Fetal del Instituto Nacional Materno Perinatal entre el 02 de enero y el 31 de diciembre del 2012, para realización de ecografía obstétrica por sospecha de acretismo.

Las pacientes fueron evaluadas aleatoriamente por cuatro médicos gineco-obstetras con entrenamiento en ultrasonido de III nivel en equipos Voluson E8 y Voluson Expert. Todas fueron evaluadas por vía abdominal inicialmente y luego se complemento la evaluación con ecografía transvaginal. El uso del modo Doppler y 3D quedó a elección del médico responsable del informe.

Se revisaron las historias clínicas de 89 de las 93 pacientes referidas para descarte de placenta acreta. Se anotaron datos de la vía del parto así como de los reportes operatorios y anatomo patológicos.

Los datos fueron registrados en una base de datos elaborada en la hoja de cálculo del programa SPSS 11,0 y los análisis descriptivos fueron realizados con el apoyo de los paquetes estadísticos EPIINFO 6 y SPSS 11,0

\section{RESULTADOS}

Durante el año 2012 se realizaron un total de 8550 evaluaciones ecográficas obstétricas en la UMF de ellas 93 fueron para descartar acretismo y 22 tuvieron diagnostico ecográfico de acretismo placentarioLa revisión de las historias clínicas descartó la sospecha de Acretismo en 66 de 78 casos (especificidad 84.6\%), y confirmó el diagnóstico ecográfico en 10 de 11 casos (sensibilidad $90.9 \%$ ) (Tabla 1) El valor predictivo positivo fue de $45 \%(10 / 22)$ y el valor predictivo negativo $99 \%$ (66/67) El porcentaje de pérdida de datos fue de $4 \%$ ya que no se pudieron ubicar 4 historias clínicas. La prevalencia real de acretismo según e presente estudio fue de 118 por cada 1000 ecografías dirigidas. La edad gestacional al momento del diagnóstico ecográfico fue (Tabla 2) en el $50 \%$ de casos entre las 32 y 36 semanas, siendo el diagnóstico más precoz el realizado a las 25 semanas.

En el $100 \%$ de los casos el diagnóstico ecográfico de acretismo estuvo asociado al hallazgo de placenta previa. Solo en un caso $50 \%$ se diagnosticó percretismo el cual fue confirmado en el acto quirúrgico requiriendo reparación quirúrgica de la vejiga. Hubo un caso adicional de percretismo el cual no fue diagnosticado prenatalmente requiriendo reparación vesical durante la cesárea (Tabla 3).

$70 \%$ de los casos terminaron en cesárea + histerectomía y en solo $30 \%$ se pudo conservar el útero mediante la sutura del lecho sangrante con puntos transfixiantes.

Tabla 1. Correlación clínica y ecográfica de acretismo placentario en los casos confirmados al nacimiento.

\begin{tabular}{lccc}
\hline Hallazgo & $\begin{array}{c}\text { Acretismo } \\
\text { confirmado }\end{array}$ & $\begin{array}{c}\text { Acretismo } \\
\text { descartado }\end{array}$ & Total \\
\hline Ecografia sugestiva & 10 & 12 & 22 \\
Ecografia normal & 1 & 66 & 67 \\
Total & 11 & 78 & 89 \\
\hline
\end{tabular}

Tabla 2. Edad gestacional al momento de diagnóstico ecográfico de acretismo placentario en los casos confirmados al nacimiento

\begin{tabular}{lcc}
\hline Edad gestacional & $\mathbf{N}^{\mathbf{0}}$ & $\%$ \\
\hline$<32$ semanas & 3 & 30 \\
$32-36$ semanas & 5 & 50 \\
$\geq 37$ semanas & 2 & 20 \\
Total & 10 & 100 \\
\hline
\end{tabular}

\section{DISCUSIÓN}

La ecografía constituye uno de los métodos para el diagnóstico prenatal del acretismo placentario junto con la Resonancia Magnética Nuclear y los marcadores biológicos (DNA fetal libre, RNAm placentario). Debido a que en nuestro medio la RMN es poco accesible por su costo y complejidad y no se cuenta con marcadores biológicos disponibles en la práctica clínica, el ultrasonido en sus diferentes formas constituye la forma principal de diagnóstico prenatal de esta condición lo cual permite la óptima planificación del manejo previniendo la morbilidad materna severa y la mortalidad mediante la disponibilidad de sangre para transfusión, así como la disponibilidad de urólogo para reparación vesical.

El diagnóstico por ecografía puede realizarse mediante ultrasonido bidimensional, Doppler y 3D. Los hallazgos ecográficos descritos asociados al acretismo van a depender del trimestre en el cual se realiza la pesquisa. En el primer trimestre tenemos ${ }^{7}$ :

1. Implantacion baja del saco gestacional

2. Áreas anecoicas placentarias

3. Interfase miometrio-placentaria anormal

En el segundo trimestre los hallazgos son más floridos describiéndose $^{8}$

1. Adelgazamiento $(<1 \mathrm{~mm})$ de la zona miometrial hipoecoica normal en el segmento inferior anterior 
Tabla 3. Características clínicas y ecográficas de las pacientes con diagnóstico confirmado de acretismo placentario

\begin{tabular}{|c|c|c|c|c|c|c|}
\hline Caso & $\begin{array}{l}\text { EG al } \\
\text { diag }\end{array}$ & $\begin{array}{l}\text { EG al } \\
\text { nacim }\end{array}$ & Parto & Hallazgos ecograficos & Observac & $\begin{array}{c}\text { Cirugia / } \\
\text { Dias hospitaliz }\end{array}$ \\
\hline 1 & $31 \mathrm{~s}$ & $32 \mathrm{~s}$ & Cesarea & $\begin{array}{l}\text { Placenta previa (Pp) total. Signos ecograficos } \\
\text { de acretismo placentario. Longitud de cervix } \\
\text { de } 18 \mathrm{~mm}\end{array}$ & $\begin{array}{l}\text { Pp total acreta sutura de } \\
\text { lecho placentario sangrante }\end{array}$ & $\begin{array}{l}\text { Cesarea / } \\
30 \text { dias }\end{array}$ \\
\hline 2 & $27 \mathrm{~s}$ & $36 s$ & $\begin{array}{l}\text { Cesarea } \\
+ \text { HAT }\end{array}$ & $\begin{array}{l}\text { Placenta previa total. Signos de acretismo pla- } \\
\text { centario }\end{array}$ & $\begin{array}{l}\text { Pp total acreta shock } \\
\text { hipovolemico }\end{array}$ & $\begin{array}{l}\text { Ces + HAT / } \\
15 \text { dias }\end{array}$ \\
\hline 3 & $33 \mathrm{~s}$ & $35 s$ & $\begin{array}{l}\text { Cesarea } \\
+ \text { HAT }\end{array}$ & $\begin{array}{l}\text { Placenta previa total con signos de acretismo } \\
\text { placentario }\end{array}$ & $\begin{array}{l}\text { Pp total acreta sangrado a } \\
\text { nivel del lecho }\end{array}$ & $\begin{array}{l}\text { Ces + HAT / } \\
8 \text { dias }\end{array}$ \\
\hline 4 & $34 \mathrm{~s}$ & $33 \mathrm{~s}$ & $\begin{array}{l}\text { Cesarea } \\
+ \text { HAT }\end{array}$ & Placenta previa total con signos de acretismo & $\begin{array}{l}\text { Pp percreta con infiltración } \\
\text { vesical. Lesión de cupula } \\
\text { vesical de } 4 \times 2 \mathrm{~cm}\end{array}$ & $\begin{array}{l}\text { Ces + HAT / } \\
13 \text { dias }\end{array}$ \\
\hline 5 & $36 s$ & $40 \mathrm{~s}$ & $\begin{array}{l}\text { Cesarea } \\
+ \text { HAT }\end{array}$ & $\begin{array}{l}\text { Placenta previa total con signos de acretismo } \\
\text { placentario }\end{array}$ & Pp total. Acreta & $\begin{array}{l}\text { Ces + HAT / } \\
13 \text { dias }\end{array}$ \\
\hline 6 & $35 \mathrm{~s}$ & $38 s$ & $\begin{array}{l}\text { Cesarea } \\
+ \text { HAT }\end{array}$ & $\begin{array}{l}\text { Placenta previa: se observa cordon umbilical } \\
\text { por delante de la presentacion en relacion a } \\
\text { vasa previa. Se observa signos ecograficos de } \\
\text { probable acretismo placentario }\end{array}$ & $\begin{array}{l}\text { Pp total. Placenta acreta. } \\
\text { Lesion vesical accidental }\end{array}$ & $\begin{array}{l}\text { Ces + HAT / } \\
7 \text { dias }\end{array}$ \\
\hline 7 & $39 \mathrm{~s}$ & $39 \mathrm{~s}$ & $\begin{array}{l}\text { Cesarea } \\
+ \text { HAT }\end{array}$ & $\begin{array}{l}\text { Placenta previa con acretismo placentario. } \\
\text { Signos de infiltracion vascular hacia la vejiga } \\
\text { y hacia el cervix }\end{array}$ & Pp acreta & $\begin{array}{l}\text { Ces + HAT / } \\
3 \text { dias }\end{array}$ \\
\hline 8 & $37 s$ & $38 s$ & $\begin{array}{l}\text { Cesarea } \\
+ \text { HAT }\end{array}$ & $\begin{array}{l}\text { Placenta previa total con signos de acretismo } \\
\text { placentario. Vasos infiltran pared posterior de } \\
\text { la vejiga en } 90 \% \text { y de cervix en } 10 \%\end{array}$ & $\begin{array}{l}\text { Pp percreta con infiltracion } \\
\text { vesical. Lesion de cupula } \\
\text { vesical de } 5 \mathrm{~cm}\end{array}$ & $\begin{array}{l}\text { Ces + HAT / } \\
4 \text { dias }\end{array}$ \\
\hline 9 & $25 s$ & $39 \mathrm{~s}$ & Cesarea & $\begin{array}{l}\text { Placenta previa total con signos de acretismo } \\
\text { placentario }\end{array}$ & $\begin{array}{l}\text { Pp total. Acreta sutura } \\
\text { de lecho placentario con } \\
\text { puntos transfixiantes }\end{array}$ & $\begin{array}{l}\text { Cesarea / } \\
3 \text { dias }\end{array}$ \\
\hline 10 & $34 \mathrm{~s}$ & $37 s$ & Cesarea & $\begin{array}{l}\text { Placenta previa centro total, al flujo doppler se } \\
\text { evidencia vasos retroplacentarios en relación } \\
\text { a mucosa vesical, sin embargo impresiona } \\
\text { que no la llega a invadir: riesgo leve a mode- } \\
\text { rado de acretismo placentario }\end{array}$ & $\begin{array}{l}\mathrm{Pp} \text { total. Acreta sutura } \\
\text { de lecho placentario con } \\
\text { puntos transfixiantes }\end{array}$ & $\begin{array}{l}\text { Cesarea / } \\
3 \text { dias }\end{array}$ \\
\hline
\end{tabular}

Nota: El caso 11 no fue diagnosticado ecográficamente.

Leyenda: HAT: Histerectomía abdominal total. Pp: Placenta previa. S: semanas. EG: Edad gestacional

2. Presencia de espacios vasculares lacunares dentro del parénquima placentario (imágen en queso suizo)

3. Adelgazamiento, irregularidad o disrupción de la interfase hiperecoica entre las paredes uterinas y la vesical

4. Extensión del tejido placentario más allá de la serosa uterina

La adición del modo Doppler color agrega nuevos hallazgos aunque no mejora sustancialmente la capacidad diagnóstica ${ }^{9,10}$, entre estos hallazgos tenemos:

1. Flujo lacunar intraparenquimal difuso o local

2. Lagos vasculares con flujo turbulento

3. Hipervascularidad de la interfase serosa - vejiga

4. Complejo venoso subplacentario prominente

Finalmente el empleo del ultrasonido tridimensional, según algunos autores, mejora notablemente tanto la sensibilidad como la especificidad ${ }^{10}$. Los hallazgos descritos por ecografía 3D comprenden:

1. Vascularización intraplacentaria irregular con vasos confluentes tortuosos que atraviesan la placenta

2. Hipervascularidad de la interfase serosa uterina-pared vesical.
La sensibilidad promedio reportada en la literatura internacional para el diagnóstico ecográfico de acretismo placentario es de $75 \%$, con valores que van desde 33 hasta $93 \%{ }^{11}$. En nuestro trabajo se hallo una sensibilidad de $90.9 \%$, lo cual es comparable a lo reportado internacionalmente.

La vía de abordaje de elección es la transvaginal, requiriendo vejiga a media repleción para adecuada visualización de la interfase vejiga-miometrio. La vía abdominal tiene menor sensibilidad y especificidad y además presenta resolución subóptima en pacientes obesas.

El segundo y tercer trimestre son los momentos en los cuales se diagnostica el acretismo con mayor frecuencia ${ }^{12}$, tal cual como se muestra en nuestro estudio donde los 10 casos confirmados fueron diagnosticados a partir de las 25 semanas; sin embargo existen reportes de la literatura en los cuales se señala que habrían signos ecográficos detectables desde el primer trimestre.

En conclusión, el ultrasonido es una herramienta útil en el diagnóstico prenatal de acretismo placentario. Debe 
sospecharse en toda paciente con diagnóstico ecográfico de placenta previa.

\section{Financiamiento: Autofinanciado.}

Conflicto de interés: El autor declara no tener algún conflicto de interés.

\section{REFERENCIAS BIBLIOGRÁFICAS}

1. Guleria K, Gupta B, Agarwal S, Suneja A, Vaid N, Jain S. Abnormally invasive placenta: changing trends in diagnosis and management. Acta Obstet Gynecol Scand. 2013; 92:461-464.

2. Wortman A, Alexander J. Placenta Accreta, Increta, and Percreta. Obstet Gynecol Clin N Am. 2013;40:137-154.

3. Wu S, Kocherginsky M, Hibbard JU. Abnormal placentation: twenty-year analysis. Am J Obstet Gynecol. 2005;192(5):1458-61.

4. Asato M. Acretismo placentario: estudio realizado durante los años 1980-1986 en el Hospital Maternidad de Lima. Tesis UPCH. Facultad de Medicina Alberto Hurtado; 1987:36pp.

5. Ayala F, Pereda J. Placenta acreta: Correlación clinicopatológica. Diagnóstico. 1992 (29): 71-74.

6. Bejar J. Histerectomía de emergencia durante la gestación, en el Instituto Materno Perinatal: Incidencia y Factores Asociados (Enero 1999 - Diciembre 2000). Tesis UNMSM. Facultad de Medicina San Fernando.
7. Ballas J, Pretorius D, Hull AD, et al. Identifying sonographic markers for placenta accreta in the first trimester. J Ultrasound Med. 2012; 31:1835

8. Wong $\mathrm{H}$, Cheung $\mathrm{Y}$, Zuccollo J, Tait J, Pringle K. Evaluation of Sonographic Diagnostic Criteria for Placenta Accreta. J Of Clinical Ultrasound. 2008; 36(9): 551-9.

9. Chou M, Ho E, Lee Y. Prenatal diagnosis of placenta previa accreta by transabdominal color Doppler ultrasound. Ultrasound Obstet Gynecol. 2000; 15: 28-35.

10. Wong $\mathrm{H}$, Cheung $\mathrm{Y}$, Williams E. Antenatal ultrasound assessment of placenta/myometrial involvement in morbidly adherent placenta. Australian and New Zealand Journal of Obstetrics and Gynaecology. 2012; 52: 67-72.

11. Esakoff TF1, Sparks TN, Kaimal AJ, Kim LH, Feldstein VA, Goldstein RB, Cheng YW, Caughey AB. Diagnosis and morbidity of placenta accreta. Ultrasound Obstet Gynecol. 2011;37(3):324-7.

12. Resnik R. Clinical features and diagnosis of placenta accreta, increta, and percreta. 2013 UpToDate. Accesed 07/07/2018.

\section{Correspondencia:}

Dirección: Av. Guardia Civil 715. San Borja

Correo electrónico: erasmohuertas@hotmail.com

Teléfono: 999144288 